\title{
FORMULATION AND EVALUATION OF DASATINIB LOADED SOLID LIPID NANOPARTICLES
}

\section{YASMIN BEGUM 1 , PRATHYUSHA REDDY GUDIPATI ${ }^{2}$}

\author{
${ }^{1}$ Assistant Professor, King Khalid University, Abha, Kingdom of Saudi Arabia, ${ }^{2}$ Student, Malla Reddy College of Pharmacy, Hyderabad, India \\ Email: yaminimp47@gmail.com
}

Received: 27 May 2018 Revised and Accepted: 27 Oct 2018

\begin{abstract}
Objective: The aim of present work was to formulate and evaluate Dasatinib (DST) loaded solid lipid nanoparticles (SLNs) as a potential anticancer drug delivery system by enhancing its solubility.
\end{abstract}

Methods: SLNs consist of a solid lipid matrix where the drug was incorporated. Surfactants of GRAS grade were used to avoid aggregation and to stabilize the SLNs. DST-SLNs formulations of varying concentrations were prepared by high-speed homogenization technique and evaluated for drug excipients compatibility study, poly-dispersity index, particle size analysis, surface morphology, zeta potential, and drug release features.

Results: It was observed that DST-SLNs with optimum quantities of poloxomer: lecithin ratio showed $88.06 \%$ drug release in $6 \mathrm{~h}$ with good entrapment efficiency of $76.9 \pm 0.84 \%$. Particle size, Polydispersity index, zeta potential and drug entrapment efficiency for the optimized formulation was found to be optimum. Stability studies revealed that the entrapment efficiency of the SLN dispersion stored in $4{ }^{\circ} \mathrm{C}$ was stable.

Conclusion: Thus, it can be concluded that the formulations of DST loaded SLNs are suitable carriers for improving the solubility and dissolution related problems.

Keywords: Dasatinib, Solid lipid nanoparticles, Lecithin, Poloxomer 188, Homogenization

(C) 2018 The Authors. Published by Innovare Academic Sciences Pvt Ltd. This is an open access article under the CC BY license (http://creativecommons.org/licenses/by/4.0/) DOI: http://dx.doi.org/10.22159/ijpps.2018v10i12.27567

\section{INTRODUCTION}

Although a large number of new drug molecules with varied therapeutic potentials have been discovered in the recent decade, yet most of them are still in the developmental process. This can be attributed to the limited aqueous solubility which governs the bioavailability of such drug molecules. One amongst them is Dasatinib (DST). Hence, there is a requisite for a technology-based product (formulation) in order to overcome such issues without compromising on the therapeutic response. DST is very poorly soluble in water [1], and maximum solubility in plain water is estimated to be about $10 \mu \mathrm{M}$ [2]. Low aqueous solubility and poor dissolution of DST results into poor and variable bioavailability [13]. Oral bioavailability of DST is ranging from $14 \%$ to $34 \%$ [3]. Poor drug solubility makes it very difficult to perform high-throughput screening of products for potential drug effects. Therefore, there is an urgent need for intelligent drug formulations to achieve sufficient bioavailability of DST. Many different approaches have been developed to overcome the solubility problem of poorly soluble drugs including solubilization, inclusion compounds and complexation. An alternative to these methods developed was drugnanoparticle formulation.

The emerging field of nanotechnology seeks to exploit distinct technological advantages of nanoscience. The booming nanotechnologies are supported by massive state investments in many countries. Companies worldwide are producing consumer products with growing "nano"-content. Significant effort has been devoted to develop biocompatible nanosystems such as nanoparticles, nanocapsules, micellar systems, and conjugates. The basic advantage of nanonization is the increase in surface area and the concentration gradient of these poorly soluble compounds followed by an increased dissolution rate of the compounds according to the Noyes-Whitney equation. In addition, the saturation solubility is also increased after nanonization [4]. All these may benefit oral bioavailability of poorly soluble drugs by enhancing drug transport through gut wall into the systemic circulation [5].

SLNs have been reported as an alternative drug delivery system to traditional polymeric nanoparticles (Mu“ hlen et al. 1998; Mehnert and Mader 2001). Nanoparticles are in the submicron size range
(50-1000 nm) and are composed of physiologically tolerated lipid components; at room temperature, the particles are in the solid state (Schwarz et al. 1993; Mu“ ller and Lucks 1996). SLNs combine the advantages of poly-meric nanoparticles, fat emulsions and liposomes (Schwarz and Mehnert 1999). They can be produced on a large industrial scale by high-pressure homogenization (Dingler and Gohla 2002), are toxicologically highly acceptable like emulsions and liposomes, produce sustained release due to their solid matrix, similar to polymeric nanoparticles, and can effectively target specific tissues after parenteral administration (Cavalli et al. 1999; Yang et al. 1999). In this study, SLNs were employed to investigate the enhancement of the absorption and bioavailability of poorly soluble drugs.

SLNs have been reported as an alternative drug delivery system to traditional polymeric nanoparticles (Mu“ hlen et al. 1998; Mehnert and Mader 2001). Nanoparticles are in the submicron size range (50-1000 nm) and are composed of physiologically tolerated lipid components; at room temperature, the particles are in the solid state (Schwarz et al. 1993; Mu“ ller and Lucks 1996). SLNs combine the advantages of polymeric nanoparticles, fat emulsions, and liposomes (Schwarz and Mehnert 1999). They can be produced on a large industrial scale by high-pressure homogenization (Dingler and Gohla 2002), are toxicologically highly acceptable like emulsions and liposomes, produce sustained release due to their solid matrix, similar to polymeric nanoparticles, and can effectively target specific tissues after parenteral administration (Cavalli et al. 1999; Yang et al. 1999). In this study, SLNs were employed to investigate the enhancement of the absorption and bioavailability of poorly soluble drugs. SLNs have been reported as an alternative drug delivery system to traditional polymeric nanoparticles $\left(\mathrm{Mu}^{*}\right.$ hlen et al. 1998; Mehnert and Mader 2001). Nanoparticles are in the submicron size range (50-1000 $\mathrm{nm}$ ) and are composed of physiologically tolerated lipid components; at room temperature, the particles are in the solid state (Schwarz et al. 1993; Mu“ ller and Lucks 1996). SLNs combine the advantages of polymeric nanoparticles, fat emulsions and liposomes (Schwarz and Mehnert 1999). They can be produced on a large industrial scale by high-pressure homogenization (Dingler and Gohla 2002), are toxicologically highly acceptable like emulsions and liposomes, produce sustained release due to their solid matrix, similar to polymeric nanoparticles, and can effectively target specific 
tissues after parenteral administration (Cavalli et al. 1999; Yang et al. 1999). In this study, SLNs were employed to investigate the enhancement of the absorption and bioavailability of poorly soluble drugs.

Solid lipid nanoparticles (SLNs) have been reported as an alternative drug delivery system to traditional polymeric nanoparticles. Nanoparticles are in the submicron size range $(50-1000 \mathrm{~nm})$ and are composed of physiologically tolerated lipid components, and at room temperature, the particles are in the solid state. SLNs combine the advantages of polymeric nanoparticles, fat emulsions, and liposomes. They can be produced on a large industrial scale by highpressure homogenization. SLNs are toxicologically highly acceptable like emulsions and liposomes, produce sustained release due to their solid matrix, similar to polymeric nanoparticles, can effectively target specific tissues after parenteral administration and found to be the effective tool in improving the oral bioavailability of poorly soluble drugs [6, 7]. In this study, SLNs were employed to investigate the enhancement of the solubility and dissolution behaviour of the poorly water-soluble drug, DST as well as DST loaded SLNs may be exploited as targeted delivery systems that can provide higher specificity to cancer cells with no/minimal effect on normal cells.

\section{MATERIALS AND METHODS}

\section{Materials}

DST was obtained as gift sample from Aizant laboratories, Hyderabad. Stearic acid, ethanol, chloroform, DMSO were obtained from SD Fine Chemicals Pvt Ltd, Mumbai. Lecithin and poloxomer 188 were obtained from Dr, Reddy's Laboratories, Hyderabad as gift samples. All the other ingredients used were of analytical grade.

\section{An analytical method for DST}

Accurately weighed quantity of DST was dissolved in little of DMSO followed by $\mathrm{pH} 4$ Acetate buffer and further dilutions were done using $\mathrm{pH} 4$ Acetate buffer to get the standard working solutions of 2 to 9 $\mu \mathrm{g} / \mathrm{ml}$. The solutions were measured with absorbance using UV spectrophotometer at $326 \mathrm{~nm}$ against the blank. Calibration curve was constructed by plotting the graph between absorbance vs. concentration.

\section{Preparation of DST loaded SLNs (homogenization)}

DST Loaded SLNs was prepared by making an organic phase containing the drug, stearic acid, and lecithin at varying ratios into ethyl alcohol $(10 \mathrm{ml}, 93 \% \mathrm{~V} / \mathrm{V})$. The aqueous phase was prepared by dissolving variable amounts of poloxamer 188 in water $(50 \mathrm{ml})$.

The organic phase was dropped into the aqueous phase at a constant rate under homogenization at $800 \mathrm{rpm}$ for $15 \mathrm{~min}$ using a high sheer homogenizer. The resulting suspension was immediately transferred to cold distilled water $(50 \mathrm{ml})$ and kept in an ice bath while stirring at $800 \mathrm{rpm}$ for $1 \mathrm{~h}$. Then the suspension was centrifuged at $5000 \mathrm{rpm}$ for $1 \mathrm{~h}$. The precipitate was washed twice with double-distilled water and suspended in mannitol solution $(10 \% \mathrm{w} / \mathrm{v})$. The suspension was lyophilized and kept at a constant temperature of $4{ }^{\circ} \mathrm{C}$ for further use [8].

Table 1: Composition of DST loaded SLNs

\begin{tabular}{|c|c|c|c|c|c|c|c|c|c|c|}
\hline S. No. & Ingredients (mg) & F1 & F2 & F3 & F4 & F5 & F6 & F7 & F8 & F9 \\
\hline 1 & Dasatinib & 10 & 10 & 10 & 10 & 10 & 10 & 10 & 10 & 10 \\
\hline 2 & Lecithin & 50 & 50 & 50 & 50 & 25 & 12.5 & 25 & - & 25 \\
\hline 3 & Poloxomer 188 & 1 & 2 & 3 & 4 & 3 & 3 & 2 & 3 & - \\
\hline 4 & Stearic acid & 100 & 100 & 100 & 100 & 100 & 100 & 100 & 100 & 100 \\
\hline 5 & Ethanol(ml) & 4 & 4 & 4 & 4 & 4 & 4 & 4 & 4 & 4 \\
\hline 6 & Chloroform & 6 & 6 & 6 & 6 & 6 & 6 & 6 & 6 & 6 \\
\hline 7 & Distilled water & q. $s$ & q. $s$ & q. $s$ & q.s & q. s & q. s & q. $s$ & q. s & q.s \\
\hline
\end{tabular}

\section{Preformulation studies}

\section{Solubility studies}

The solubility of DST was determined in various solvents such as acetone, acetonitrile, ethanol, methanol, DMSO, and chloroform using the shake flask method.

\section{Evaluation of DST loaded SLNs \\ FTIR compatibility studies}

FTIR spectrum was recorded over the region of 400 to $4000 \mathrm{~cm}^{-1}$ for pure drug alone and along with excipients in BRUKER FTIR Instrument using $\mathrm{KBr}$ pellet technique.

\section{Determination of zeta potential}

The Zeta Master apparatus (Malvern Instruments, Malvern.) was used to calculate zeta potential by determining the electrophoretic mobility of the optimized formulation. It was obtained by performing an electrophoresis experiment on the sample and measuring the velocity of particles. There exists an electrical double layer at the interface between solid particle surface and the surrounding liquid medium. The inner electrical layer is tightly bound to the surface whereas the outer layer is loosely attached due to electrostatic forces and Brownian motion developed. The glycerosomal particle moves in the liquid medium along with its associated charges as a unit. The ionic potential at the surface between this unit and the surrounding medium is called as Zeta potential, and it is measured in millivolts [9].

\section{Particle size and morphology}

Scanning electron microscopy was used to verify the uniformity of particle shape and size [9]. The lyophilized nanoparticles were dropped onto double-sided carbon tape. They were then vacuumcoated for 45 seconds with a platinum mixture and examined for morphology with a FE-SEM (JEOL JSM7500, Thermo Scientific) at $5 \mathrm{kV}$.

\section{Drug entrapment efficiency}

The percentage of drug entrapped was determined by centrifugation method. $10 \mathrm{ml}$ of aqueous suspension was centrifuged at 10,000 rpm for 20 min. The amount of drug was detected in the supernatant and in the pellet. The entrapment efficiency was calculated using the following formula [10]

EntrapmentEf ficiencyPercentage $=$ Entrappeddrug $/$ Totaldrug $X 100$

\section{In vitro diffusion study}

In vitro release studies were carried out by using modified diffusion assembly USP XXI. The Semipermeable membrane was conditioned by soaking in PH4 Acetate buffer for $24 \mathrm{~h}$. The donor compartment consists of a glass tube with an inner diameter of $2.5 \mathrm{~cm}$, open at both ends and tied at with dialysis membrane. The DST loaded SLNs, equivalent to 10 mg of DST was taken in this compartment and placed in a beaker containing $250 \mathrm{ml}$ of $\mathrm{pH} 4$ acetate buffer, which was magnetically stirred at moderate speed $(200 \mathrm{rpm})$ at a temperature of $37^{\circ} \mathrm{C} .2 \mathrm{ml}$ sample was withdrawn and same volume of medium was replaced at predetermined interval and was assayed UV Spectrophotometrically at $326 \mathrm{~nm}$ against $\mathrm{pH} 4$ acetate buffer as blank. The in vitro release profiles of these formulations were then compared with the pure drug [11].

\section{Stability studies}

Stability study was conducted for the formulation F5 having high entrapment efficiency by storing the formulation at two different temperatures, $4{ }^{\circ} \mathrm{C}$ and $25 \pm 2{ }^{\circ} \mathrm{C}$ and the drug content was estimated every $15 \mathrm{~d}$, to find out any change in the entrapment efficiency of the SLNs [12].

\section{RESULTS AND DISCUSSION}

\section{Solubility studies}

The solubility of DST was found to be $200 \mathrm{mg} / \mathrm{ml}$ in DMSO. It was found to be poorly soluble in water and ethanol. So DMSO was 
FTIR compatibility studies

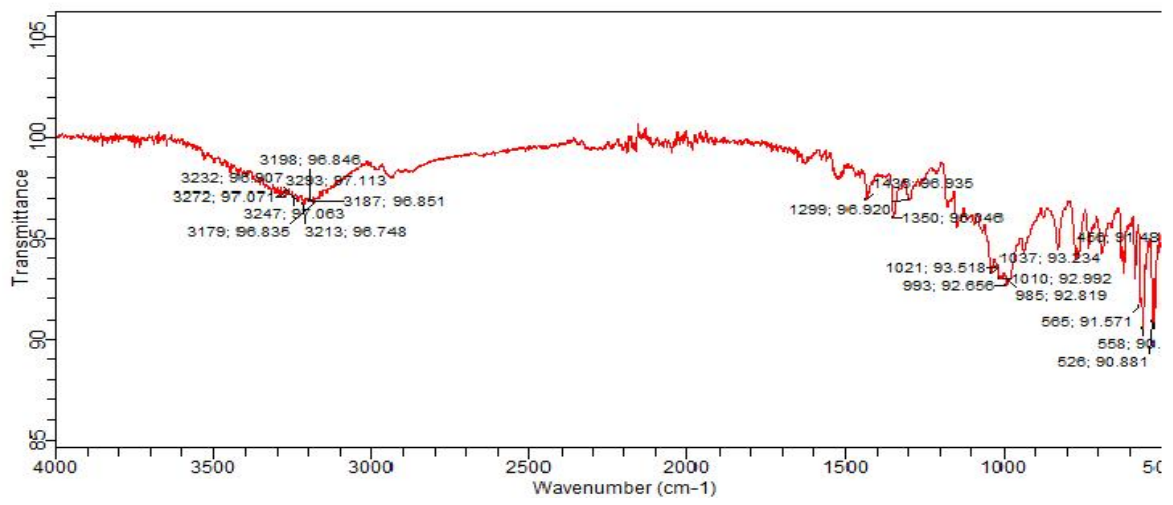

Fig. 1: FTIR spectra of DST

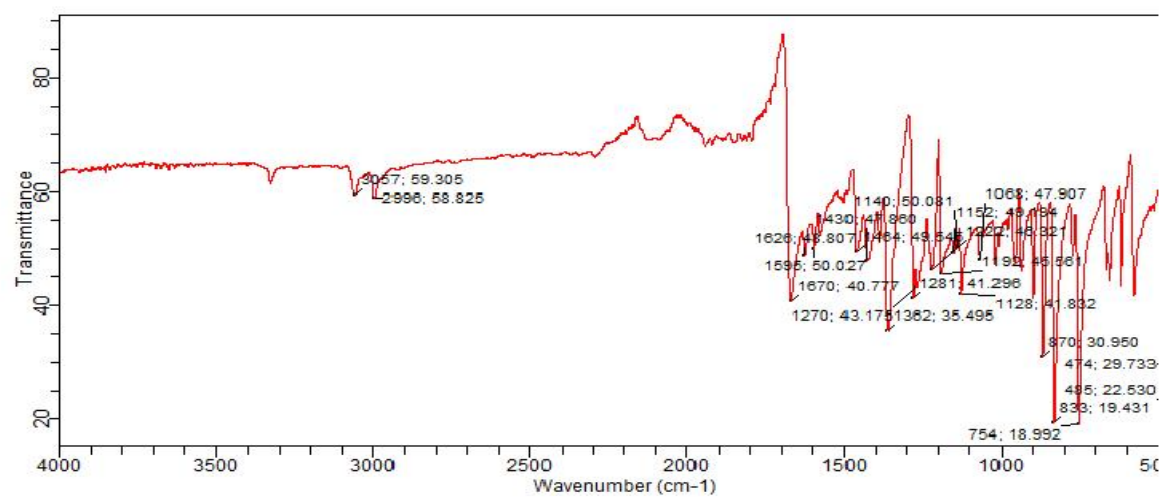

Fig. 2: FTIR spectra of DST along with excipients

Table 2: FTIR compatibility studies

\begin{tabular}{llll}
\hline Groups & \multicolumn{2}{l}{ FTIR significant peaks } & \\
\cline { 2 - 4 } & Reported values & Observed values for pure drug (cm-1) & Observed values for drug and excipients (cm-1) \\
\hline C-C stretching & $1300-400$ & $1362.54 \mathrm{~cm}-$ & $1281.84 \mathrm{~cm}-1$ \\
C=O stretching & $1700-1600$ & $1436.15 \mathrm{~cm}-1$ & $1626.73 \mathrm{~cm}-1$ \\
C-N stretching & $1080-1360$ & $1192.64 \mathrm{~cm}-1$ & $1068.93 \mathrm{~cm}-1$ \\
N-H stretching & $3600-3310$ & $3179 \mathrm{~cm}-1$ & $3057.59 \mathrm{~cm}-1$ \\
Aromatic C-H stretching & $3200-3000$ & $3187 \mathrm{~cm}-1$ & $2996.58 \mathrm{~cm}-1$ \\
Aromatic C-H bending & $1000-675$ & $993.92 \mathrm{~cm}-1$ & $870.30 \mathrm{~cm}-1$ \\
C-Cl stretching & $600-800$ & $485.32 \mathrm{~cm}-1$ & $558.90 \mathrm{~cm}-1$ \\
\hline
\end{tabular}

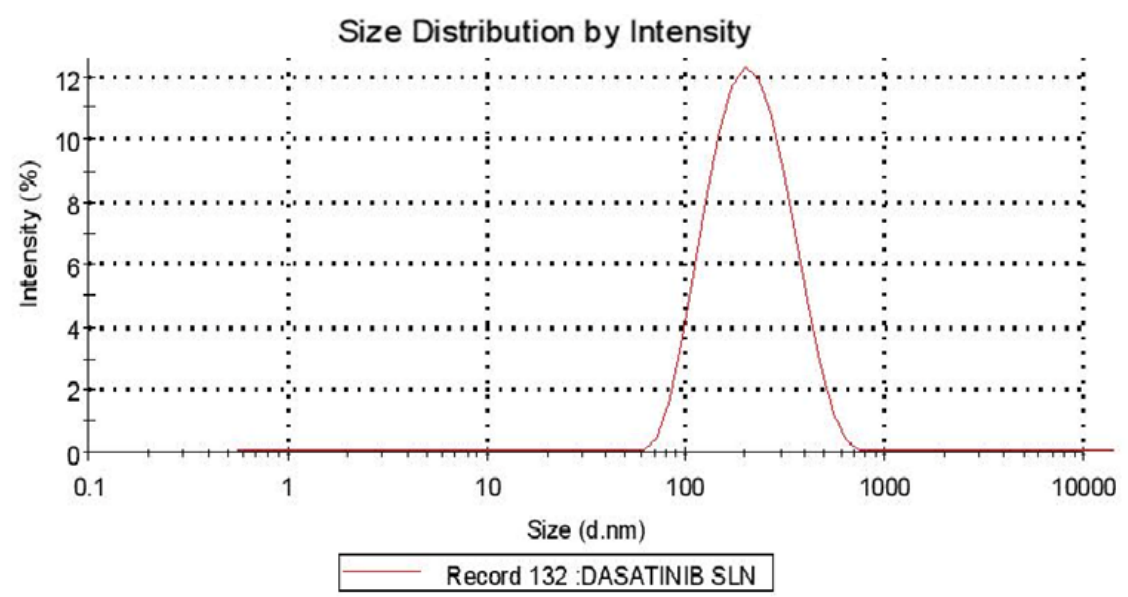

Fig. 3: Particle size distribution of DST loaded SLNs 
FT-IR spectroscopic study was carried out to find out drug excipients interaction. The characteristic peaks of the drug between IR spectrum of DST and drug excipients mixture were identified by absorption peaks at $3179 \mathrm{~cm}-1$ (secondary amine N-H stretch), 3187 $\mathrm{cm}-1$ (=C-H aromatic ring), $993.92 \mathrm{~cm}-1$ ( $\mathrm{C}-\mathrm{H}$ bending), $1192.64 \mathrm{~cm}-$ 1 (C-N stretch), $1436 \mathrm{~cm}-1$ (C=0 stretch) and $1362 \mathrm{~cm}-1$ (C=C) stretch, aromatic ring. Thus, FTIR Spectroscopy was employed to obtain conformational information about the lipid molecules, and it is used to investigate the interactions between lipid, drug and excipients thereby confirming that there were no particular interactions between them and drug remain intact in the formulation. The principal peaks of DST were observed in the spectra of the drug and lipids physical mixture indicating no interactions had been occurred [13].

\section{Particle size analysis}

It measures and relates the size of particles typically in the submicron region by Brownian motion. (Brownian motion is the random movement of particles due to the bombardment by the solvent molecules that surrounds them). Particle size depends on the hardness of the drug, process parameters, and materials used such as stabilizers, emulsifying agents, etc. The analysis revealed that the SLN produced had a particle size ranging from $94 \mathrm{~nm}$ to 800 $\mathrm{nm}$ with a polydispersity index ranging from 0.11 to 0.44 .

\section{Measurement of zeta potential}

The zeta potential of nanoparticles is commonly used to characterize the surface charge property of nanoparticles. It reflects the electrical potential of particles and is influenced by the composition of the particle and the medium in which it is dispersed. Optimized formula f5 had good zeta potential values thereby indicating the optimized formulation had comparatively effective stability.

The zeta values of all the formulations were from-67.90 to $+31.75 \mathrm{mV}$. Thus, the range of zeta potential obtained was found to be high enough for sufficient electrostatic stabilization. However, the wide range of values may be due to the shielding effect of poloxamer 188 which is at varying concentration, and this acts as a cover for the surface charges due to the presence of its hydrophilic corona around the nanoparticles. Since poloxamer 188 may offer additional steric stabilization for SLN, it is assumed that there was a combination of electrostatic and steric stabilization in the investigated SLN formulae.

They showed the formation of small nanoparticles with relatively low polydispersity indices. Although there was an insufficient charge to stabilize the nanoparticles electrostatically, there was steric stabilization due to the added poloxamer [9].

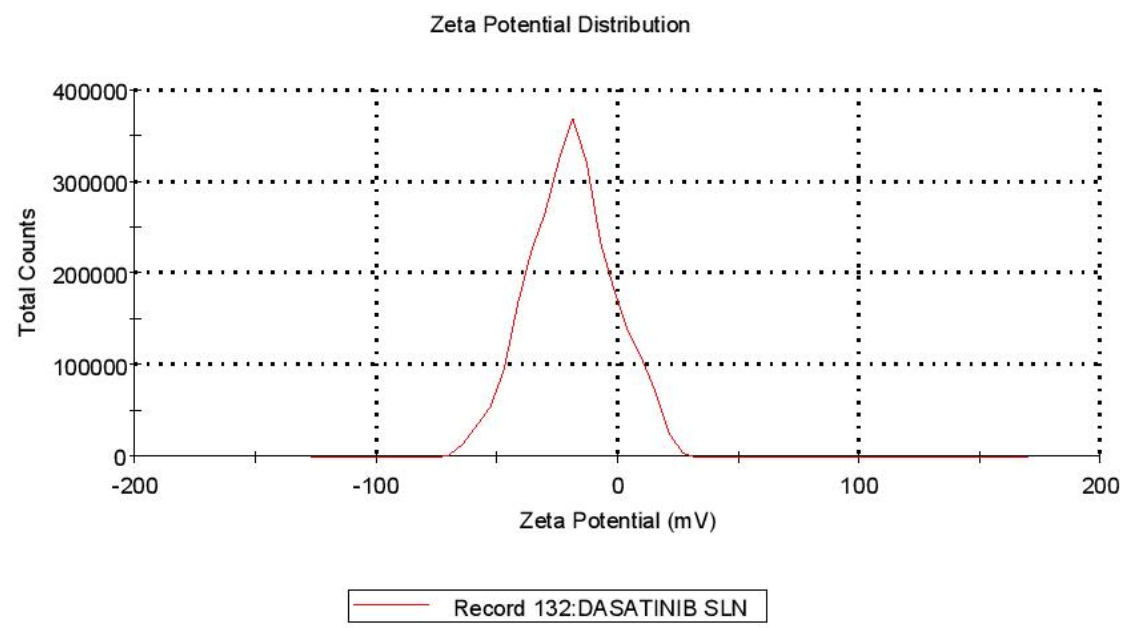

Fig. 4: Zeta potential of DST loaded SLNs

Table 3: Results of particle size analysis and zeta potential

\begin{tabular}{llll}
\hline Formulation code & Average particle size (nm) & Polydispersity index(PI) & Zeta potential(mV) \\
\hline F1 & 309.84 & 0.17 & 31.23 \\
F2 & 340.89 & 0.29 & 39.65 \\
F3 & 365.52 & 0.21 & 32.98 \\
F4 & 400.02 & 0.17 & 47.90 \\
F5 & 202.60 & 0.18 & 31.75 \\
F6 & 188.45 & 0.11 & -33.75 \\
F7 & 94.58 & 0.19 & -66.16 \\
F8 & 708.53 & 0.23 & -67.90 \\
F9 & 800.36 & 0.44 & -30.23 \\
\hline
\end{tabular}

\section{Scanning electron microscopy}

The particles have moderate uniformity and all the particles were discrete entities, shows aggregation of particles after lyophilization and these particles was readily re-dispersible. It was clear from the image that DST loaded SLNs were near spherical in shape with the presence of some particle aggregates. This is particularly because of the particle being coated with a surfactant layer.

\section{Drug entrapment efficiency}

The maximum encapsulation efficiency of $76.9 \%$ was found to be for F5 containing the optimum amounts of lecithin and poloxamer.
The encapsulation efficiency was found to below $20.34 \%$ and $15.86 \%$ respectively for the formulations that did not contain either lecithin (F8) or poloxamer (F9). The combined effect of the two independent variables, namely poloxamer 188 (which was measured as a percentage) and lecithin (which was measured in milligrams), were studied in respect of the encapsulation efficiency. The results revealed that increasing the concentration of poloxamer 188 had the dominant effect of increasing the encapsulation efficiency. It is clear from the data that the encapsulation efficiency is directly proportional to the concentration of the poloxamer 188, however, this is only the case when mixed with lecithin. 


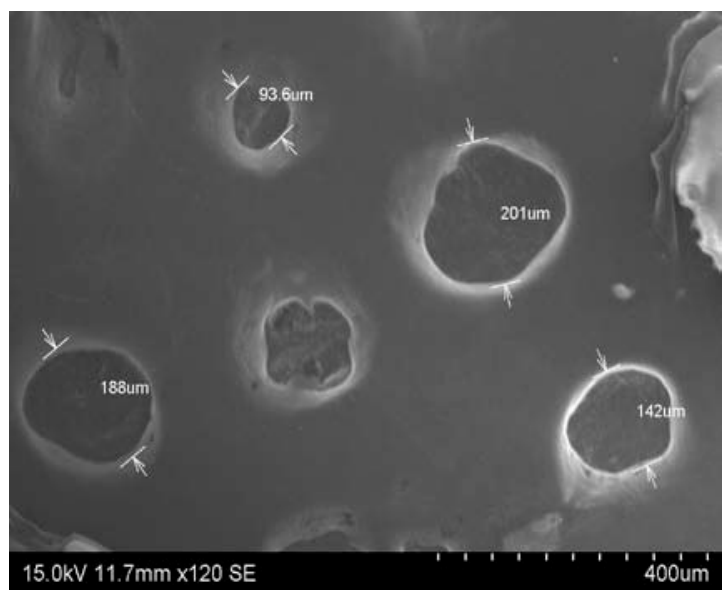

Fig. 5: SEM for optimized formulation

Table 4: Entrapment efficiency of DST loaded SLNs

\begin{tabular}{ll}
\hline Formulation code & Entrapment efficiency (\%) \\
\hline F1 & $42.37 \pm 1.94$ \\
F2 & $54.12 \pm 0.53$ \\
F3 & $62.89 \pm 0.96$ \\
F4 & $56.26 \pm 1.3$ \\
F5 & $76.9 \pm 0.84$ \\
F6 & $63.28 \pm 0.81$ \\
F7 & $46.18 \pm 0.34$ \\
F8 & $20.34 \pm 1.9$ \\
F9 & $15.86 \pm 1.52$ \\
\hline
\end{tabular}

Data are represented as mean \pm standard deviation (SD), $n=3$

Using poloxamer188 without lecithin failed to increase the encapsulation efficiency as measured for F8. Conversely, increasing the concentration of lecithin, in the presence of poloxamer188, increased the encapsulation efficiency. The results indicate that both poloxamer and lecithin played a pivotal role in the encapsulation efficiency of the drug. However, the concentration of poloxamer was more significant in its effects on encapsulation efficiency than lecithin. The poloxamer may assist to form the hydrophilic shell surrounding the SLN and thus enhances its stability. Lecithin has high lipophilicity and is a water-insoluble surfactant. In the present study, the addition of poloxamer to the aqueous medium led to the formation of a mesh-type network that surrounded the particles and prevented the drug from migrating into the aqueous medium. However, as proven by the encapsulation efficiency results obtained in the absence of poloxamer, the importance of poloxamer with its capabilities to entrap the drug within the shell region and to prevent their escape was revealed. Our hypothesis postulates that the fraction of drug that entered the water when the poloxamer was absent is relatively large. Here, we stress the importance of poloxamer in stabilizing the SLNs, in addition to the prevention of escape of the reversed drug from the SLNs [8].

\section{In vitro drug release study}

The results showed that the increase in the concentration of the surfactant increase drug release from the SLN until an optimum concentration after that the drug release was found to be decreased. The formulation F5 prepared using optimum amount of lipid and surfactant was found to show higher drug release of $88.06 \%$ in 6hours. When the amount of lecithin was reduced to $50 \%$ it did not make any considerable differences in drug release. But the further reduction in lecithin concentration reduced the drug entrapment efficiency as well as drug release (F6). Formulations prepared without lecithin (F8) and without poloxamer 188 (F9) showed a drastic reduction in drug entrapment efficiency as well as drug release.

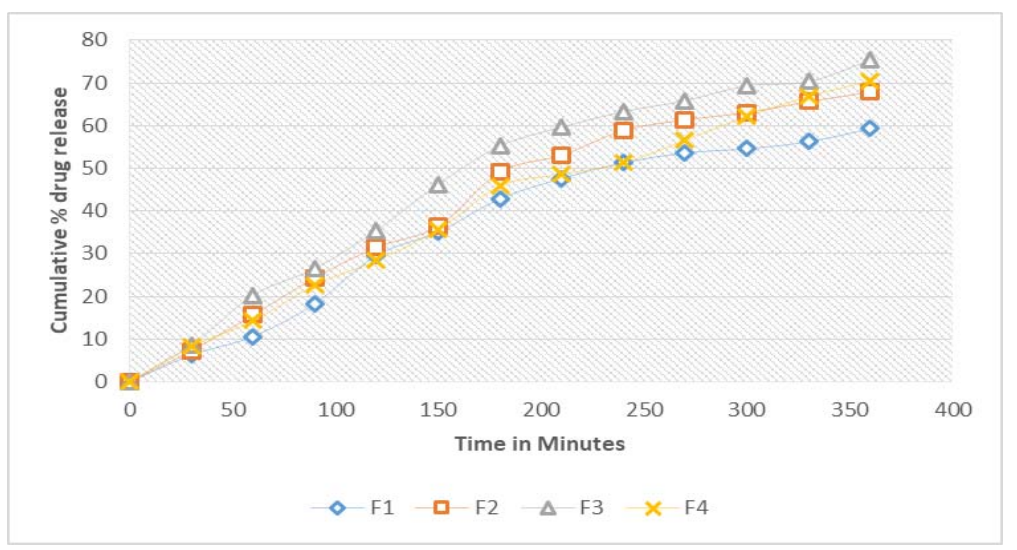

Fig. 6: Drug release profile of DST loaded SLNs for formulations from F1 to F4 (n=3; mean \pm SD) 


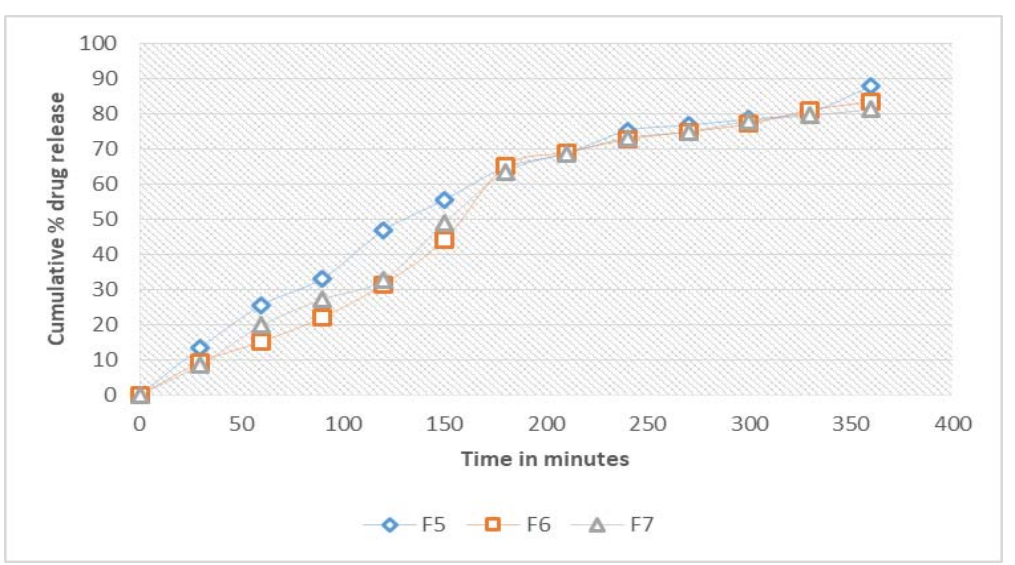

Fig. 7: Drug release profile of DST loaded SLNs for formulations from F5 to F7 ( $n=3$; mean \pm SD)

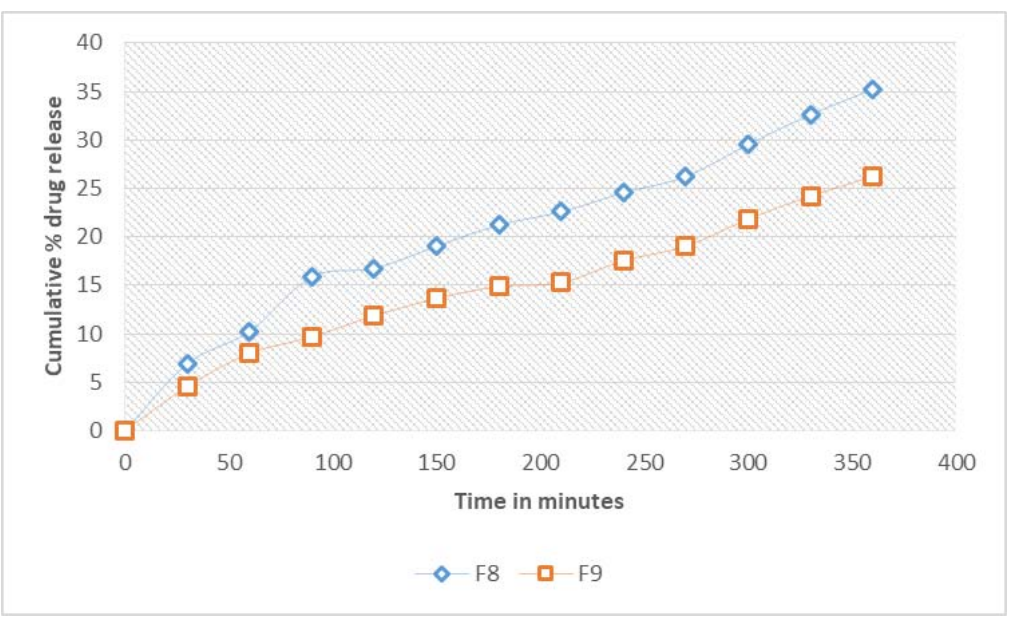

Fig. 8: Drug release profile of DST loaded SLNs for formulations F8 and F9 (n=3; mean \pm SD)

\section{Stability studies}

The results revealed that the entrapment efficiency of the SLN dispersion stored in $4{ }^{\circ} \mathrm{C}$ was found to be stable when compared to the SLN dispersion stored in $25{ }^{\circ} \mathrm{C} \pm 2{ }^{\circ} \mathrm{C}$. This may be due to more drug expulsion from the lipid matrices at a higher temperature.

Table 5: Results of stability studies of the formulation F5

\begin{tabular}{lllll}
\hline Formulation & \% Drug encapsulation efficiency & & \\
\cline { 2 - 5 } & Immediately after preparation & $\mathbf{1 5}^{\text {th }}$ Day & & \\
\hline Temperature & & $4^{\circ} \mathrm{C}$ & $25^{\circ} \mathrm{C} \pm 2{ }^{\circ} \mathrm{C}$ & $4^{\circ} \mathrm{C}$ \\
F5 & $76.9 \pm 0.84$ & $76.2 \pm 0.67$ & $74.9 \pm 0.72$ & $75.3 \pm 0.51$ \\
\hline
\end{tabular}

Data are represented as mean \pm standard deviation (SD), $\mathrm{n}=3$.

\section{CONCLUSION}

SLNs have emerged as a promising drug delivery system in recent years as its potential is gradually and eventually being realized. Many drugs having therapeutic activity are highly lipophilic in nature. Incorporation and delivery of such lipophilic active ingredients at the drug delivery site/target site has always been a concern. It was observed that the homogenization and ultrasound dispersion method was a useful method for the successful incorporation of the poorly water-soluble drug DST with high entrapment efficiency. Furthermore, it could be presumed that if the nanometer range particles were obtained, the bioavailability might be increased. Hence, we can conclude that SLNs are used as drug carriers for lipophilic drugs, to enhance the bioavailability of poorly water-soluble drugs through nanoparticles, as a drug delivery system.

\section{AUTHORS CONTRIBUTIONS}

All the authors contributed equally in making the manuscript ready for submission.

\section{CONFLICT OF INTERESTS}

Authors declare no conflict of interest

\section{REFERENCES}

1. Rakesh P, Nitish T. Studies in development of dasatinib nanoformulations. Eur J Pharm Med Res 2016;3:423-32.

2. Saikat R, Rosalynn Q, Adam JM. Structural and physicochemical aspects of dasatinib hydrate and anhydrate phases. Cryst Growth Des 2012;12:2122-6.

3. Siu FW. New dosing schedules of dasatinib for CML and adverse event management. J Hematol Oncol 2009;2:10-6. 
4. Sandeep K, Vijaykumar N. A review on improved delivery of poorly soluble compounds using nanoparticle technology. Drug Delivery Transl Res 2016;6:319-32.

5. Lee J. Nanoparticle formulation increases oral bioavailability of poorly soluble drugs: approaches experimental evidences and theory. Curr Nanosci 2005;1:237-43.

6. Lian Dong H, Xing T, FuDe C. Solid lipid nanoparticles (SLNs) to improve oral bioavailability of poorly soluble drugs. J Pharm Pharmacol 2004;56:1257-535.

7. Singh H, Gupta RD, Gautam G. Formulation development, characterization, and in vitro-in vivo study of antihyperlipidemic drug rosuvastatin calcium-solid lipid nanoparticles. Asian J Pharm Clin Res 2018;11:436-43.

8. Rasha A, Khallaf, Heba F, Salem, Ahmed A. 5-Fluorouracil shellenriched solid lipid nanoparticles (SLN) for effective skin carcinoma treatment. Drug Delivery 2016;23:3452-60.
9. Nandini PT, Doijad RC, Shivakumar HN, Dandagi PM. Formulation and evaluation of gemcitabine-loaded solid lipid nanoparticles. Drug Delivery 2015;22:647-51.

10. Srawan GY, Madhuri K. Formulation and evaluation of clozapine solid lipid nanoparticles with natural lipid. Drug Discovery Ther 2014;2:18-26.

11. Senthil P, Arivuchelvan A, Jagadeeswaran A, Subramanian N, Senthil C, Mekala P. Formulation, optimization and evaluation of enrofloxacin solid lipid nanoparticles for sustained oral delivery. Asian J Pharm Clin Res 2015;8:231-6.

12. Ekambaram $P$, Hasan $S$, Abdul A. Formulation and evaluation of solid lipid nanoparticles of ramipril. J Young Pharm 2011;3:216-20.

13. Abather A, Sadiq A, Abdul R. Formulation and evaluation of silibinin loaded solid lipid nanoparticles for peroral use targeting lower part of gastrointestinal tract. Int J Pharm Pharm Sci 2014;6:55-67. 